\title{
Etiological Diagnosis of Community-Acquired Pneumonia in Adult Patients: A Prospective Hospital-Based Study in Mashhad, Iran
}

\author{
Hamidreza Naderi ${ }^{1}$; Fereshte Sheybani ${ }^{1, *}$; Mohammadreza Sarvghad ${ }^{1}$; Zahra Meshkat ${ }^{2}$; \\ Mehdi Jabbari Nooghabi ${ }^{3}$ \\ ${ }_{1}^{1}$ Department of Infectious Diseases, Imam Reza Teaching Hospital, Faculty of Medicine, Mashhad University of Medical Sciences, Mashhad, IR Iran \\ ${ }^{2}$ Department of Clinical Microbiology and Virology, Faculty of Medicine, Mashhad University of Medical Sciences, Mashhad, IR Iran \\ ${ }^{3}$ Department of Statistics, Faculty of Mathematics, Ferdowsi University, Mashhad, IR Iran \\ ${ }^{*}$ Corresponding author: Fereshte Sheybani, Department of Infectious Diseases, Imam Reza Teaching Hospital, Faculty of Medicine, Mashhad University of Medical Sciences, Mashhad, \\ IR Iran. Tel: +98-9151254062, Fax: +98-5138407742, E-mail: fereshtesheybani@gmail.com; sheybanif@mums.ac.ir
}

Received: August 14, 2014; Revised: October 22, 2014; Accepted: November 10, 2014

\begin{abstract}
Background: Pneumonia is the third most common cause of death in the world, and mortality is highest for patients who require hospitalization.

Objectives:This prospective observational study is an etiological survey of community-acquired pneumonia (CAP) over a 12-month period in the Iranian city of Mashhad. To our knowledge, this is one of the first prospective hospital-based studies to comprehensively evaluate the epidemiological, demographical, clinical, and prognostic factors of patients with CAP in Iran.

Patients and Methods:We studied all adult patients (aged $\geq 16$ years) with CAP admitted to Imam Reza Hospital, Mashhad, Iran, between February 2013 and January 2014. The etiological diagnosis of CAP was made through conventional culturing and staining of respiratory secretions (i.e. sputum and pleural fluid), standard ВACTEC ${ }^{\text {M }}$ Plus Aerobic/F bottles for blood cultures, and the immunochromatographic assays BinaxNOW ${ }^{\circledR}$ Streptococcus pneumoniae antigen and BinaxNOW ${ }^{\circledR}$ Legionella pneumophila antigen for the detection of S. pneumoniae antigen and $L$. pneumophila serogroup 1 antigen, respectively.

Results:Among 120 patients with CAP, the most common etiology was S.pneumoniae (24.4\%), followed by Mycobacterium tuberculosis(17.5\%), S. aureus (6.7\%), polymicrobial agents including anaerobes (4.2\%), complicated hydatid cyst (2.5\%), Influenza A virus ( $4.2 \%$; including 2 cases of mixed Influenza A-bacterial infection), and Klebsiella pneumoniae, Brucella melitensis, Mucor, and varicella, each in $0.8 \%$ of the patients. The diagnosis of pneumonia remained unknown in 49 (40\%) patients.

Conclusions: Tuberculosis was an important cause of CAP in our region. Hence, it should be considered in all patients admitted with a CAP diagnosis.
\end{abstract}

Keywords: Pneumonia; Etiology; Tuberculosis; Streptococcus pneumoniae

\section{Background}

In 1901, Sir William Osler noted in the fourth edition of his book, the principles and practice of medicine, that "the most widespread and fatal of all acute diseases, pneumonia, is now Captain of the Men of Death." Over a century later, the prominence of pneumonia as a clinical entity remains (1). Nowadays, it is the third most common cause of death in the world accounting for $7 \%$ of the total mortality of 56 million people. According to The world health organization (WHO) data, 3 - 4 million people die due to pneumonia, a large proportion of whom are children or elderly (2). Mortality is highest for CAP patients who require hospitalization, with a 30-day mortality rate of up to $23 \%$ in such patients. It has also been shown that all-cause mortality in patients with community-acquired pneumonia (CAP) is as high as $28 \%$ within one year (3).

It is often difficult to determine the microbiological cause of pneumonia. A microbiological diagnosis is confirmed in about $60 \%$ of the cases of CAP in research studies that use specialized tests to detect various pathogens but in only about $20 \%$ of the cases in everyday practice (4). Despite geographical variations, Streptococcus pneumoniae is the most common cause of pneumonia worldwide (4). The rank order of the most common causes of pneumonia varies according to the severity of the illness. Therefore, when considering the etiology of CAP, it is useful to categorize patients into those who can be treated on an ambulatory basis, those who require hospitalization, and those who require admission to an intensive care unit (ICU) (5). There is a dearth of research on the etiology and prevalence of CAP in Iran. Our review of the published and unpublished literature to identify studies on CAP in Iran yielded only a few studies that evaluated the etiological diagnosis of CAP in adults, most of them including only one or two pathogens.

\section{Objectives}

The present prospective observational study is an etio-

Copyright (c) 2015, Ahvaz Jundishapur University of Medical Sciences. This is an open-access article distributed under the terms of the Creative Commons Attribution-NonCommercial 4.0 International License (http://creativecommons.org/licenses/by-nc/4.0/) which permits copy and redistribute the material just in noncommercial usages, provided the original work is properly cited. 
logical survey of CAP during a period of 12 months in the Iranian city of Mashhad. To our knowledge, this is one of the first prospective hospital-based studies to comprehensively evaluate the epidemiological, demographical, clinical, and prognostic factors of patients with CAP in Iran.

\section{Patients and Methods}

The study was prospective and observational.

\subsection{Selection and Description of Participants}

We included in this study nearly all adult patients (aged $\geq 16$ years) admitted to Imam Reza Hospital, a 1000-bed university hospital in Mashhad, Iran, between February 2013 and January 2014, with the diagnosis of CAP. The exclusion criteria comprised history of transplantation, recent chemotherapy with/without iatrogenic neutropenia within the previous 3 months, pneumonia caused by hospitalization or within the previous 3 months, chronic pneumonia ( $>4$ weeks), and refusal to sign the informed consent form. Out of 166 patients with community-acquired lower respiratory tract involvement with chest infiltrates, 140 cases were eligible for this study. Twenty patients with lower respiratory symptoms and infiltrates on chest radiography with an emerging alternative/noninfectious diagnosis during the follow-up (e.g. pulmonary emboli or malignancy) were secondarily excluded.

Statistical analyses were only performed on the remaining 120 patients with the diagnosis of acute CAP with an infectious etiology after the secondary exclusion. Clinical, radiological, and microbiological data of the patients were collected. Elderly was defined as persons aged $\geq 65$ years. Chronic obstructive pulmonary disease (COPD) was defined clinically as the presence of a chronic productive cough for $\geq 3$ months during each of 2 consecutive years (other causes of cough being excluded). Bedridden status was defined as confined to bed by sickness or old age. Opium addiction was defined by behaviors that included one or more of the following: impaired control over drug (opioid) use; compulsive use; continued use despite harm; and craving. The patients gave written informed consent, and the study was approved by the Ethics Committee of Mashhad University of Medical Sciences (Code: 900983).

\subsection{Technical Information}

The diagnostic criteria for CAP included an infiltrate on chest radiograph in a patient with either fever or clinical signs/symptoms of the lower respiratory tract infection, or both. Pneumonia was defined as acute if the symptoms lasted $<4$ weeks. Parapneumonic effusion was defined as exudative pleural effusion associated with lung infection, i.e. pneumonia. According to Light's traditional criteria, if at least one of the following three criteria was present, the fluid was defined as exudates:

A. Pleural fluid protein/serum protein ratio > 0.5;

B. Pleural fluid lactate dehydrogenase (LDH)/serum LDH ratio $>0.6$; and
C. Pleural fluid LDH > two-thirds the upper limits of the laboratory's normal serum LDH.

The sputum specimens were sent to the microbiological laboratory and processed immediately. No special procedures were performed to obtain sputum samples if they could not be obtained spontaneously except for endotracheal aspiration for the patients with early endotracheal intubation. A Gram stain was performed on a purulent portion of each sputum specimen. The sputum samples were considered of good quality if they had $<10$ squamous cells and $>25$ leukocytes per low-power field. Otherwise, the sputum sample was considered contaminated by saliva and rejected. The good quality specimens were then screened for a predominant bacterial morphological type in an oil-immersion field. Only the good quality specimens were used to culture the bacteria. The sputum specimens were also stained using Ziehl-Neelsen staining for the detection of mycobacteria. Blood for culture was inoculated in standard BACTEC ${ }^{\mathrm{TM}}$ Plus Aerobic/F bottles (Becton Dickinson, Ireland).

The unconcentrated urine samples were tested with the immunochromatographic assays BinaxNOW ${ }^{\circledR}$ S. pneumoniae antigen (Binax, Maine, U.S.A.) and BinaxNOW ${ }^{\circledR}$ Legionella pneumophila antigen (Binax, Scarborough, Maine, U.S.A.) for the detection of S. pneumoniae antigen and L. pneumophila serogroup 1 antigen, respectively. Legionella spp. was evaluated by urinary antigen testing only, but not by culture or polymerase chain reaction (PCR).

In our study, diagnostic tests for Mycoplasma pneumoniae, Chlamydophila pneumoniae, Chlamydophila psittaci, and Coxiella burnetii were not used. Viral etiology was not evaluated except for influenza. In the case of varicella pneumonia, the presumptive diagnosis was based on the compatible clinical and radiological manifestations of pneumonia and the presence of characteristic vesicular rash. PCR for the influenza virus was performed on nasopharyngeal swab specimens only in the patients with compatible clinical and epidemiological findings with or without a bacterial etiology confirmed by rapid tests, reverse transcription polymerase chain reaction (RT-PCR) with the Qiagen one-step real-time. The detection kit was used for the purification and detection of the influenza virus. The procalcitonin (PCT) levels were determined using a semi-quantitative solid-phase immunoassay (B.R.A.H.M.S. PCT-Q, B.R.A.H.M.S.-Diagnostica GmbH, Hennigsdorf, Germany) on $200 \mu \mathrm{L}$ plasma. The PCT levels were categorized into four groups (i.e. $<0.5 \mu \mathrm{g} / \mathrm{L}, 0.5-<2$ $\mu \mathrm{g} / \mathrm{L}, 2-<10 \mu \mathrm{g} / \mathrm{L}$, and $\geq 10 \mu \mathrm{g} / \mathrm{L}$ ) according to the provided reference scale. The test was performed within the first 12 hours of patient admission.

The etiology of CAP was classified as definitive if one of the following criteria was met: 1. Identification of an etiological agent from blood or pleural fluid; 2 . Detection of the S. pneumoniae antigen or L. pneumophila antigen in urine; 3. Detection of the influenza virus from a nasopharyngeal sample by PCR; and 4. One or more initial sputum smear examinations positive for acid-fast bacilli 
Naderi H et al.

(AFB) or positive PCR for Mycobacterium tuberculosis on body fluids. $M$. tuberculosis DNA was extracted from the clinical samples using an M. tuberculosis Real-Time PCR Kit (Rotor-Gene ${ }^{\mathrm{TM}} 3000$ (Corbett Life Science)).

The etiology of pneumonia was defined as presumptive when the identification of the etiological agent was based on: 1. Predominant organism in a good quality sputum sample Gram staining (defined as an average of $>10$ organisms of proper structure per oil-immersion field, $>75 \%$ of organisms on the slide of proper structure, or when either of these two criteria was met) and a positive sputum culture. 2. Patients in whom the Gram staining of the sputum revealed a mixed flora with the accompanying foul smell of the sputum were defined as having presumptive aspiration pneumonia with polymicrobial agents, including anaerobic organisms. 3. Clinical, laboratory, and radiological findings compatible with pulmonary tuberculosis with no response to the empirical antibiotic therapy and other etiological agents as the cause of clinical syndrome were ruled out; but M. tuberculosis was not isolated.

\subsection{Statistics}

The statistical analyses were performed using Statistical Package for the Social Sciences (SPSS) and the R programming language. The discrete variables are expressed as counts (percentage) and the continuous variables as mean \pm standard deviation (SD), unless stated otherwise. Frequency comparison was done via the $\chi^{2}$ test or the Fisher exact test for the categorical variables and the Student t-test or the Mann-Whitney U test for the continuous variables. A P value $<0.05$ was considered significant.

\section{Results}

The 120 patients with the diagnosis of acute CAP with an infectious etiology were comprised of 76 (63.3\%) males and 44 (36.7\%) females. The mean age of the study popula- tion was $50.4 \pm 22.6$ years $(17-94)$. The patients' ages were not normally distributed. More than one-third (35.8\%) of all the patients hospitalized for CAP were elderly, $46.5 \%$ of whom were $>75$ years old. The male-to-female ratio was 1.7 . However, the trend of more male subject enrolment was not seen across all the age groups. The most common clinical findings were cough in $85 \%(n=102)$ and fever in $83.3 \%$ ( $n=100)$ of the patients, followed by sputum production $52 \%(n=62)$, dyspnea $77.5 \%(n=93)$, altered mental status $32.5 \%(n=39)$, chest pain $18.3 \%(n=22)$, hemoptysis $13.3 \%$ ( $n=16)$, nausea/vomiting 13.3\% $(n=16)$, diarrhea 5.8\% ( $n$ $=7)$, myalgia $20.8 \%(n=25)$, and weight loss $15.8 \%(n=19)$. Of the 120 patients in our study, 66 (55\%) cases had classic lobar pneumonia on chest radiography. Less common radiographic findings included bronchopneumonia in 30 (25\%), cavitary lung lesion(s) in 13 (10.8\%), diffuse interstitial/reticular infiltrates in 11 (9.1\%), necrotizing infiltrates in 10 (8.3\%), micronodular infiltrates in $5(4.1 \%)$, and mixed infiltrates in 10 (8.3\%) patients. Sixty-seven (55.8\%) patients had bilateral involvement on chest radiography.

The most common underlying diseases in the patients were COPD (18.3\%), diabetes mellitus (6.6\%), and bedridden status (6.6\%). Two patients with CAP were diagnosed to have HIV infection, and one patient who presented with community acquired non-responding pneumonia of 3 weeks' duration was finally diagnosed as a case of pneumococcal pneumonia in the setting of common variable immunodeficiency. The most common etiological diagnosis was S. pneumoniae (24.4\%), followed by $M$. tuberculosis (17.5\%), S. aureus (6.7\%), polymicrobial agents including anaerobes (4.2\%), complicated hydatid cyst (2.5\%), Influenza A virus (4.2\%) (including 2 cases of mixed Influenza A-bacterial infection), and Klebsiella pneumoniae, Brucella melitensis, Mucor, and varicella, each in $0.8 \%$ of the patients. The diagnosis of pneumonia remained unknown in $49(40 \%)$ patients (Table 1$)$.

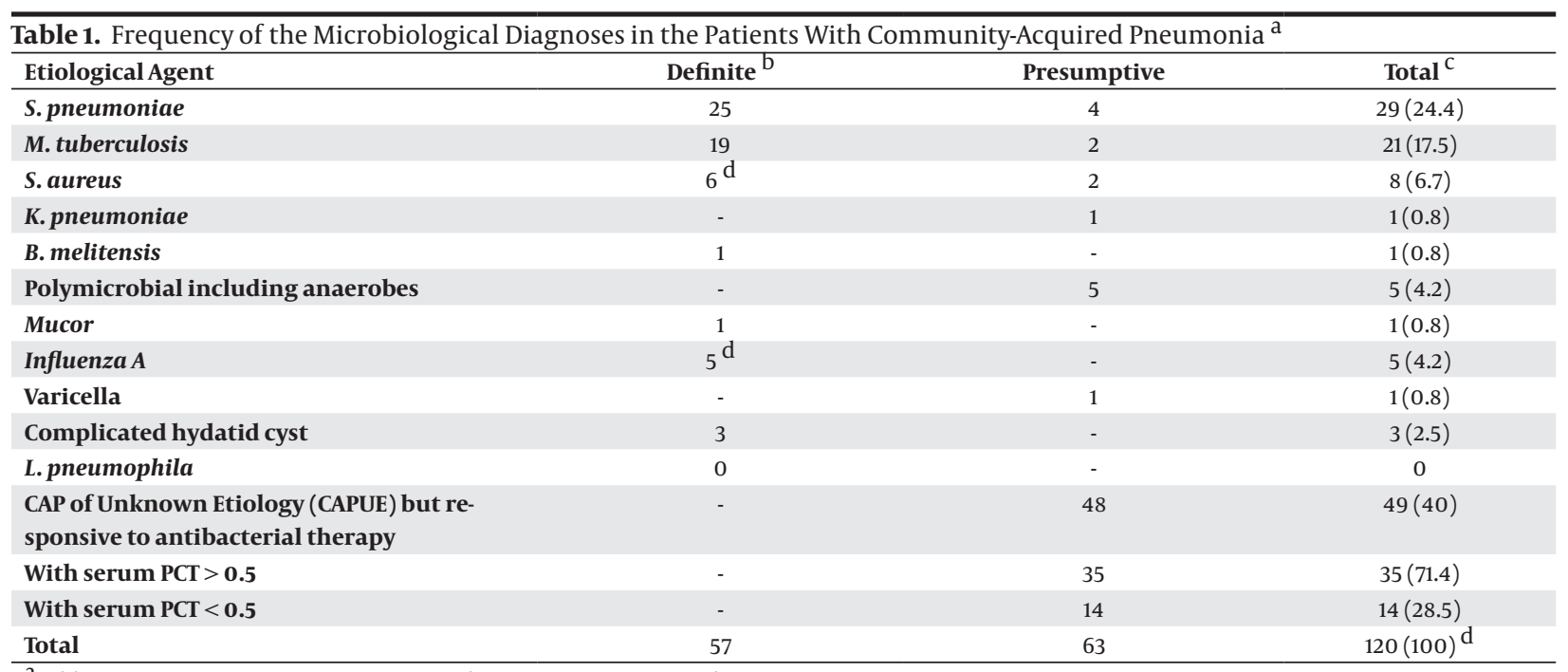

\footnotetext{
a Abbreviations: CAP: Community-Acquired Pneumonia, PCT: Procalcitonin.

b Pneumococcal CAP was diagnosed exclusively by the urinary antigen test in 9 (31\%) cases.

c Values are presented as No. (\%).

d Two cases had mixed infection with influenza virus and S. aureus.
} 
The patients with community-acquired pneumonia of unknown etiology (CAPUE) were classified into two groups: 1) CAPUE with PCT $\geq 0.5 \mathrm{ng} / \mathrm{mL}$ (71.4\%) and 2) CAPUE with PCT $<0.5 \mathrm{ng} / \mathrm{mL}$ (28.5\%). Among the patients with pulmonary tuberculosis, 3 had concomitant tuberculous meningitis, which was confirmed by positive cerebrospinal fluid PCR for $M$. tuberculosis. All of them had a miliary pattern on chest radiography. Additionally, the usefulness of the sputum Gram stain in guiding the etiological diagnosis was assessed. Sixty-one per cent of the patients had some type of respiratory specimens, including good quality sputum, endotracheal tube aspirate, and/or pleural effusion for Gram staining and culture. For the patients with the definite diagnosis of pneumococcal pneumonia (25 patients), the Gram stain suggested pneumococcal infection in 17 (65.3\%). The blood cultures were positive in 4 (3.33\%) of the 120 patients. Parapneumonic effusions were observed in $30.8 \%$ of the patients. The frequency of parapneumonic effusions was $33.3 \%$ in pneumococcal pneumonia, $62.5 \%$ in staphylococcal pneumonia, and $20.4 \%$ in the patients with CAPUE. Pleural effusion was detected in $38.1 \%$ of the patients with tuberculosis. The only case of brucella pneumonia was complicated with loculated parapneumonic effusion.

The overall in-hospital mortality (IHM) rate was $23.6 \%$. The rate was $33.3 \%$ among the elderly patients compared with the younger adults, with a $20.3 \%$ death rate, which was higher, but not statistically significant $(\mathrm{P}=0.14)$. The 3-day mortality rate was 3.3\%. Intensive vasopressor and respiratory support (IVRS) was required in 38 (36.1\%) patients, 16 (42.1\%) of whom did not receive IVRS in the first
24 hours post admission. The study population included 46 (38.7\%) opioid addicts. The frequency distribution of the etiological organisms among this group of CAP patients was as follows: S. pneumoniae 16 (34.8\%); Mycobacterium 11 (23.9\%); S. aureus 4 (8.7\%); polymicrobial agents including anaerobes 1 (2.2\%); varicella 1 (2.3\%); complicated hydatid cyst 1 (2.3\%); and CAPUE but responsive to antibacterial therapy 11 (24.5\%).

As was mentioned earlier, more than one-third of our patients were elderly. The frequency distribution of the etiological organisms among the elderly patients with CAP was as follows: S. pneumoniae 14 (32.6\%); Mycobacterium 5 (11.6\%); S. aureus 5 (11.6\%); K. pneumoniae 1 (2.3\%); polymicrobial agents including anaerobes 3 (7.1\%); mucormycosis 1 (2.4\%); Influenza A 1 (2.4\%); complicated hydatid cyst 1 (2.4\%); and CAPUE but responsive to antibacterial therapy 16 (37.3\%). The etiological diagnosis remained unknown in $37.3 \%$ of the elderly patients, which was lower than that in the younger adults, but the difference was not statistically significant (39\% vs. $49.1 \%, \mathrm{P}=0.829$ ). A comparative analysis between these two age groups revealed that the duration of clinical symptoms before admission was significantly lower ( $7.6 \pm 4.4$ vs. $11.1 \pm 7.7$ days, $\mathrm{P}=0.016)$ and the presence of underlying diseases $(\mathrm{P}<$ $0.001)$ and altered mental status on admission $(P=0.003)$ was more frequent in the elderly patients. The IHM rate and the number of the patients who needed IVRS were higher in the patients $\geq 65$ years compared with the younger adults; nevertheless, these differences were not statistically significant $(\mathrm{P}=0.063$ and 0.242 , respectively) (Table 2).

\begin{tabular}{|c|c|c|c|}
\hline Patients' Characteristics & Elderly & Young Adult & P Value \\
\hline Age, $y$ & $75.9 \pm 7.4$ & $36.1 \pm 13.1$ & 0.001 \\
\hline Duration of symptoms & $7.6 \pm 4.4$ & $11.1 \pm 7.7$ & 0.016 \\
\hline Underlying diseases & $29(67.4)$ & $25(32.9)$ & 0.001 \\
\hline Addiction & $13(30.2)$ & $33(43.4)$ & 0.156 \\
\hline Bedridden Status & $6(14)$ & $2(2.6)$ & 0.047 \\
\hline Altered mental status & $21(50)$ & $17(23)$ & 0.003 \\
\hline Hypotension & $9(23.7)$ & $14(19.7)$ & 0.62 \\
\hline Tachycardia & $31(83.8)$ & $61(84.7)$ & 0.898 \\
\hline Tachypnea & $35(92.1)$ & $61(83.6)$ & 0.212 \\
\hline High-grade fever & $26(68.4)$ & $54(76.1)$ & 0.39 \\
\hline Severe hypoxia & $17(44.7)$ & $31(43.7)$ & 0.914 \\
\hline Pleurisy & $9(29)$ & $19(30.6)$ & 0.53 \\
\hline Bilateral involvement & $18(43.9)$ & $44(62)$ & 0.064 \\
\hline IVRS requirement & $16(39)$ & $21(28.4)$ & 0.242 \\
\hline Mechanical ventilation after $24 \mathrm{~h}$ of admission & $8(19.5)$ & $8(10.7)$ & 0.187 \\
\hline In-hospital mortality & $14(33.3)$ & $14(18.2)$ & 0.063 \\
\hline
\end{tabular}

\footnotetext{
a The values are presented as mean \pm standard deviation or No (\%).
b Abbreviation: IVRS, Intensive Vasopressor or Respiratory Support.
} 


\section{Discussion}

In this study, we evaluated the frequency of the microbiological etiology of CAP in adult patients who required hospital admission during a period of 12 months. We found that $S$. pneumoniae was the most common etiological agent of CAP (24.4\%). This result chimes in with previous studies that showed this organism as the most common etiology of CAP with a frequency of $10 \%$ to $20 \%$ (6). Identifying the cause of CAP remains a challenge. Concordant with some previous studies, we could not diagnose the etiology of CAP in a significant number of the patients (40.4\%), 69.6\% of whom had PCT $>0.5 \mathrm{ng} / \mathrm{mL}$. It has been previously demonstrated that the PCT level is lower in pneumonia caused by viral and atypical pathogens than a typical bacterial etiology $(7,8)$. It can be postulated, but not documented, that $31.4 \%$ of the patients with CAPUE and PCT levels $<0.5 \mathrm{ng} / \mathrm{mL}$ might have had an atypical or viral etiology. The other causative agents among our patients included M. tuberculosis, S. aureus, polymicrobial agents including anaerobes, complicated hydatid cyst, Influenza A virus, K. pneumoniae, B. melitensis, Mucor, and varicella.

The use of blood and pleural culture in the etiological diagnosis of CAP has been hampered by the limited sensitivity. Bacterial pneumonia may have been underestimated in our study due to the low rate of the bacteremic cases and the low number of the positive cultures with a definitive diagnostic value in the non-tuberculous patients with CAP (3.33\% and 8\%, respectively). Previously, the diagnostic yield of blood culture was reported at $<10 \%$ ( 9 , 10). In a study conducted by Benenson et al. (9), three factors were associated with positive blood cultures: oxygen saturation $<90 \%$; serum sodium $<130 \mathrm{mEq} / \mathrm{L}$; and respiratory rate $>30$ breaths/minute.

The authors suggested that although the sensitivity of the pneumococcal urinary antigen test is lower in patients who are not bacteremic, the presence of a positive urinary antigen test in a non-bacteremic patient can be helpful for tailoring therapy. The main disadvantage of this test is its false-positive results in the case of nasopharyngeal colonization, especially in children or in patients with recent episodes of pneumococcal infection. However, because of the exclusion criteria of our study, this problem was not of significance.

This finding is in contrast to the results of some previous studies that demonstrated a frequency of $6 \%$ to $13 \%$ for L. pneumophila in patients with community-acquired and nosocomial pneumonia in different parts of Iran (13-18) and up to $22.1 \%$ in pregnant patients with CAP (19). Additionally, several studies have documented the contamination of different water sources with Legionella in our country (20-22). The detection of the L. pneumophila antigen by the immunochromatographic assay had optimum sensitivity for the detection of L. pneumophila serogroup 1. However, most evidence shows that L. pneumophila serogroup 1 is the etiological agent of $70 \%$ of the community-acquired Legionnaires' disease in most parts of the world $(23,24)$. Accordingly, it can be suggested that L. pneumophila is not an important and prevalent etiological cause of CAP in our region, compared to other microorganisms. In a study performed in Japan, the incidence of $L$. pneumonia was also far lower than that in Western countries (25).

The sensitivity and specificity of the sputum Gram stain vary substantially in different settings. The sensitivity of the Gram stain compared to culture ranges between $15 \%$ and $100 \%$ and its specificity ranges from $11 \%$ to $100 \%$ (11). Of the 29 patients in our study with the diagnosis of pneumococcal pneumonia, the etiological diagnosis of 8 (27.5\%) cases was only documented based on a positive $S$. pneumoniae urinary antigen test. The important role of this assay in the rapid etiological diagnosis of CAP could not be overemphasized, especially in patients who cannot produce good quality sputum, those who are not bacteremic, and those with the administration of antibiotics before admission. Sorde et al. (12) studied adult patients hospitalized with CAP caused by S. pneumoniae and diagnosed pneumococcal CAP exclusively by the urinary antigen test in $44 \%$.

The estimates of Legionnaires' disease as a cause of CAP requiring hospitalization in adults range from $0.5 \%$ to $10 \%$ of all admitted pneumonia cases; an average value is probably about $2 \%$, even in geographical regions with excellent diagnostic capabilities (1). Although we tested all the patients' urine samples with the immunochromatographic Legionella urinary antigen assay, L. pneumophila was detected by the urinary antigen in no patients in our study.

The most common underlying conditions in our patients with CAP were age $\geq 65$ years, COPD, diabetes mellitus, and bedridden status. Elderly, defined as persons aged $\geq 65$, accounted for more than one-third of all the patients hospitalized for CAP in our study. The most common organisms isolated in this age group were S. pneumoniae, M. tuberculosis, and polymicrobial agents including anaerobes, respectively. Additionally, the etiological diagnosis was not identified in $37.3 \%$ of these patients. Previously, the importance of S. pneumoniae as a cause of CAP in the elderly has been documented (26). Hashemi et al. (27) compared the frequency of the bacterial agents of CAP between elderly individuals and younger adults admitted to hospital and found that S. pneumoniae was the most frequent pathogen of CAP in the elderly patients, followed by S. aureus and Pseudomonas aeruginosa.

According to some previous studies, the mortality rate among hospitalized CAP patients is less than $15 \%$. The overall IHM rate was $23.6 \%$ in our patients, which is high compared with the previously reported rates $(28,29)$. It has been demonstrated that the first days of admission are associated with the highest mortality (29). Early mortality has been attributed to respiratory failure (30). In contrast, only $4.2 \%$ of the IHM occurred within the first 
Naderi H et al.

3 days (3-day mortality) in our study. Halm et al. (31) demonstrated that the initial severity of pneumonia correlated with the number of days until clinical stability. On the other hand, Menendez et al. (32) reported that adherence to antibiotic guidelines was associated with earlier clinical stability. Once a patient's condition becomes stable, the risk of serious clinical deterioration is $1 \%$ or less (31). In contrast, the highest incidence of IHM in our study occurred after the first day and a significant number of IVRS requirements happened after the first 24 hours of hospitalization. This finding could be a reflection of delayed clinical stability in our patients. Therefore, the difference in the distribution of hospital deaths over time in our study compared with some previous studies could have several possible explanations, including patient-related, pathogen-related, and/or management-related factors.

Among hospitalized patients, mortality rates are higher in patients with severe pneumonia, especially in those requiring treatment in an ICU. In a meta-analysis by Fine et al. (28), the mortality of the CAP patients treated in an ICU was $37 \%$. It has been mentioned that since the criteria for ICU admission vary both between hospitals and between countries, assessing IVRS rather than simple ICU admission may be more useful in evaluating the severity of pneumonia because it is probably a more objective marker of CAP severity across institutions and health care systems (33). According to some previous studies, about $10 \%-20 \%$ of hospitalized patients with CAP require IVRS (33-35), whereas more than one-third (36.1\%) of our patients needed IVRS. Accordingly, our patients presented with more severe illness. However, only $57.9 \%$ of these requirements occurred within the first 24 hours.

Mortality is also higher among elderly patients (14\% - 18\%) $(28,29,36)$. It has also been demonstrated that in the hospitalized elderly, CAP is a common and frequently fatal disease that often requires ICU admission and mechanical ventilation and consumes considerable health care resources (37). In our study, the IHM rate was 33.3\% among the elderly patients compared with the younger adults, with a $20.3 \%$ death rate, which was higher, but not statistically significant $(\mathrm{P}=0.14)$. The mean length of stay (LOS) in hospital was longer and the number of the cases who needed IVRS was higher among the elderly patients compared with the younger adults. Underlying diseases, including bedridden status, were also more common in the older patients.

In conclusion, although S. pneumoniae is the most common cause of CAP in many countries, there are considerable geographic differences in the incidence of other pathogens. Tuberculosis is an important cause of CAP in our region, and this diagnosis should be considered in all patients who present with CAP. It can also be suggested that L. pneumophila is not an important and prevalent etiological cause of CAP in our region, compared to other microorganisms. Age $\geq 65$ years accounted for approximately one-third of the patients with higher IHM, longer LOS, and more frequent underlying diseases.

\section{Acknowledgements}

This article was derived in part from a thesis (\# 2799-T) written by Fereshte Sheybani for specialty in infectious diseases. This study was approved by the ethics committee of Mashhad University of Medical Sciences (Code: 900983).

\section{Authors' Contributions}

Hamidreza Naderi, Fereshte Sheybani, Mohammadreza Sarvghad, and Zahra Meshkat contributed in designing, data gathering, writing, and editing the manuscript and Mehdi Jabbari Nooghabi contributed in designing the manuscript and statistical analysis.

\section{References}

1. Mandell GL, Bennett JE, Dolin R. Principles and Practice of Infectious Diseases. 7 edPhiladelphia: Churchill Livingstone; 2010.

2. Lopez AD, Mathers CD, Ezzati M, Jamison DT, Murray CJ. Global and regional burden of disease and risk factors, 2001: systematic analysis of population health data. Lancet. 2006;367(9524):1747-57.

3. File TJ, Marrie TJ. Burden of community-acquired pneumonia in North American adults. Postgrad Med. 2010;122(2):130-41.

4. Marrie TJ, Basow DS, Waltham MA. Uptodate; 2012. Epidemiology, pathogenesis, and microbiology of community-acquired pneumonia in adults.

5. Mandell LA, Wunderink RG, Anzueto A, Bartlett JG, Campbell GD, Dean NC, et al. Infectious Diseases Society of America/American Thoracic Society consensus guidelines on the management of community-acquired pneumonia in adults. Clin Infect Dis. 2007;44(Supplement 2):S27-72.

6. Bartlett JG. Diagnostic tests for agents of community-acquired pneumonia. Clin Infect Dis. 2011;52 Suppl 4:S296-304.

7. Hedlund J, Hansson LO. Procalcitonin and C-reactive protein levels in community-acquired pneumonia: correlation with etiology and prognosis. Infection. 2000;28(2):68-73.

8. Berg P, Lindhardt BO. The role of procalcitonin in adult patients with community-acquired pneumonia--a systematic review. Dan Med J. 2012;59(3):A4357.

9. Benenson RS, Kepner AM, Pyle D2, Cavanaugh S. Selective use of blood cultures in emergency department pneumonia patients. $J$ Emerg Med. 2007;33(1):1-8.

10. Ishida T, Hashimoto T, Arita M, Ito I, Osawa M. Etiology of community-acquired pneumonia in hospitalized patients: a 3-year prospective study in Japan. Chest.1998;114(6):1588-93.

11. Roson B, Carratala J, Verdaguer R, Dorca J, Manresa F, Gudiol F Prospective study of the usefulness of sputum Gram stain in the initial approach to community-acquired pneumonia requiring hospitalization. Clin Infect Dis. 2000;31(4):869-74.

12. Sorde R, Falco V, Lowak M, Domingo E, Ferrer A, Burgos J, et al Current and potential usefulness of pneumococcal urinary antigen detection in hospitalized patients with communityacquired pneumonia to guide antimicrobial therapy. Arch Intern Med. 2011;171(2):166-72.

13. Alavi SM, Moshiri N, Moosavian M, Yusefi F, Abbasi E. Seroprevalence of Legionella pneumophila in admitted patients with pneumonia in training hospitals, Ahvaz, Iran (2007-2008). PakJ Med Sci. 2009;25(5):811-6.

14. Fard SY, Nomanpour B, Fatolahzadeh B, Mobarez AM, DarbanSarokhalil D, Fooladi AA, et al. Hospital acquired pneumonia: comparison of culture and real-time PCR assays for detection of Legionella pneumophila from respiratory specimens at Tehran hospitals. Acta Microbiol Immunol Hung. 2012;59(3):355-65.

15. Bagheri Lankarani K, Vodjdani R, Khozaii A, Beheshti M, Oboodi B, Alborzi A, et al. Legionella pneumophillia In Southern Iran. Shiraz E-Med J. 2004;5(1).

16. Hajia M, Hossieni-Doust R, Rahbar M. Identification of Legionel- 
la Pneumophila in Bronchoalveolar Lavage Fluid Specimens by PCR. Arch Iran Med. 2004;7(4):287-91.

17. Mirkalantari S, Mohabati Mobarez A, Hoseini Dost SR, Aslani J Isolation of Legionella from BAL samples of Legionella pneumonia patients, by PCR and Culture Methods. Modares $j$ Med Sci: Pathobiology. 2008;10:75-83.

18. Nomanpour B, Ghodousi A, Babaei T, Jafari S, Feizabadi MM. Single tube real time PCR for detection of Streptococcus pneumoniae, Mycoplasma pneumoniae, Chlamydophila pneumoniae and Legionella pneumophila from clinical samples of CAP. Acta Microbiol Immunol Hung. 2012;59(2):171-84.

19. Chamani-Tabriz L, Jeddi-Tehrani M, Motahari S, Zeraati H, Asgari S, Karegar M, et al. Detection of Legionella pneumophila by nested PCR-RFLP and ELISA on urine specimens of pregnant women with respiratory infections. . 2007; 8(3):221-229. Med J Reprod Infertil. 2007;8(3):221-9.

20. Moosavian M, Dashti A. Isolation and identification of legionellosis agents from fishponds, swimming pools and cooling towers in Khuzestan province, Iran. . Jundishapur J.Microbiol. 2011;4(4):209-16.

21. Ghotaslou R, Yeganeh Sefidan F, Akhi MT, Soroush MH, Hejazi MS Detection of legionella contamination in tabriz hospitals by PCR assay. Adv Pharm Bull. 2013;3(1):131-4.

22. Ajami B, Ghazvini K, Movahhed T, Ariaee N, Shakeri M, Makarem S. Contamination of a dental unit water line system by legionella pneumophila in the mashhad school of dentistry in 2009. Iran Red Crescent Med J. 2012;14(6):376-8.

23. Den Boer JW, Nijhof J, Friesema I. Risk factors for sporadic community-acquired Legionnaires' disease. A 3-year national casecontrol study. Public Health. 2006;120(6):566-71.

24. Ricketts KD, Joseph CA, European Working Group for Legionella I. Legionnaires disease in Europe: 2005-2006. Euro Surveill. 2007;12(12):E7-8.

25. Ishida T. [Etiology of community-acquired pneumonia among adult patients in Japan].Jpn J Antibiot. 2000;53 Suppl B:3-12.

26. Jokinen C, Heiskanen L, Juvonen H, Kallinen S, Kleemola M, Koskela $\mathrm{M}$, et al. Microbial etiology of community-acquired pneumonia in the adult population of 4 municipalities in eastern Finland. Clin Infect Dis. 2001;32(8):1141-54.

27. Hashemi SH, Soozanchi G, Jamal-Omidi S, Yousefi-Mashouf R, Mamani M, Seif-Rabiei MA. Bacterial aetiology and antimicrobial re- sistance of community-acquired pneumonia in the elderly and younger adults. Trop Doct. 2010;40(2):89-91.

28. Fine MJ, Smith MA, Carson CA, Mutha SS, Sankey SS, Weissfeld LA, et al. Prognosis and outcomes of patients with communityacquired pneumonia. A meta-analysis. JAMA. 1996;275(2):134-41.

29. Ewig S, Birkner N, Strauss R, Schaefer E, Pauletzki J, Bischoff H, et al. New perspectives on community-acquired pneumonia in 388406 patients. Results from a nationwide mandatory performance measurement programme in healthcare quality. Thorax. 2009;64(12):1062-9.

30. Garcia-Vidal C, Calbo E, Pascual V, Ferrer C, Quintana S, Garau J. Effects of systemic steroids in patients with severe communityacquired pneumonia. Eur Respir J. 2007;30(5):951-6.

31. Halm EA, Fine MJ, Marrie TJ, Coley CM, Kapoor WN, Obrosky DS, et al. Time to clinical stability in patients hospitalized with community-acquired pneumonia: implications for practice guidelines. JAMA. 1998;279(18):1452-7.

32. Menendez R, Torres A, Rodriguez de Castro F, Zalacain R, Aspa J, Martin Villasclaras JJ, et al. Reaching stability in communityacquired pneumonia: the effects of the severity of disease, treatment, and the characteristics of patients. Clin Infect Dis. 2004;39(12):1783-90.

33. Charles PG, Wolfe R, Whitby M, Fine MJ, Fuller AJ, Stirling R, et al. SMART-COP: a tool for predicting the need for intensive respiratory or vasopressor support in community-acquired pneumonia. Clin Infect Dis. 2008;47(3):375-84.

34. Davis JS, Cross GB, Charles PG, Currie BJ, Anstey NM, Cheng AC. Pneumonia risk stratification in tropical Australia: does the SMART-COP score apply? Med J Aust. 2010;192(3):133-6.

35. Robins-Browne KL, Cheng AC, Thomas KA, Palmer DJ, Currie BJ, Davis JS. The SMART-COP score performs well for pneumonia risk stratification in Australia's Tropical Northern Territory: a prospective cohort study. Trop Med Int Health. 2012;17(7):914-9.

36. Marras TK, Gutierrez C, Chan CK. Applying a prediction rule to identify low-risk patients with community-acquired pneumonia. Chest. 2000;118(5):1339-43.

37. Kaplan V, Angus DC, Griffin MF, Clermont G, Scott Watson R, Linde-Zwirble WT. Hospitalized community-acquired pneumonia in the elderly: age- and sex-related patterns of care and outcome in the United States. Am J Respir Crit Care Med. 2002;165(6):766-72. 